\title{
GUT-ASSOCIATED LYMPHOID TISSUE IN MICROTUS montanus From Natural Populations
}

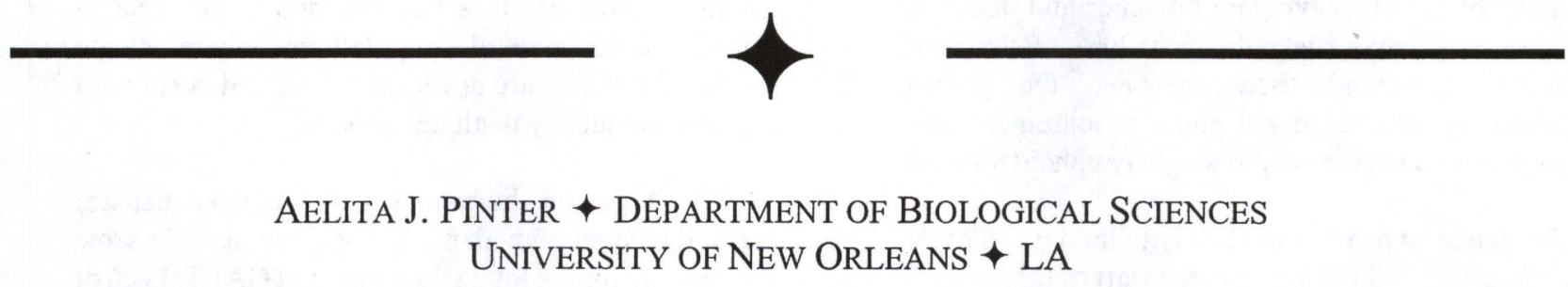

\section{$\uparrow$ OBJECTIVES}

Impaired immunocompetence may contribute to the multiannual fluctuations ("cycles") in population density of arvicoline rodents (Mihok et al. 1985, Pinter 1988, Lochmiller et al. 1993). In a variety of species environmental variables influence at least one component of the immune system, the gut-associated lymphoid system, GALT (Lopez et al. 1985, Li et al. 1995). Preliminary laboratory observations suggest that the development of GALT in Microtus montanus can be influenced by such factors as age and diet (Smith and Pinter 2000). However, these observations are from captive-bred voles, maintained on a synthetic laboratory diet, under strictly controlled laboratory conditions. The complexity and abundance of variables under natural conditions must influence the development and function of GALT - yet, nothing is known about it from Microtus montanus under natural conditions. The purpose of this study was essentially twofold: (1) to describe the morphometrics of GALT in wild-caught M. montanus, and (2) to characterize the morphometric changes that occur in GALT of these animals as a function of their age, sex, and the season of the year.

\section{$\uparrow \quad$ Methods}

The tissues examined in this study were obtained from Microtus montanus trapped for a longterm study of their population dynamics in Grand Teton National Park (see "Climatic factors, reproductive success and population dynamics in the montane vole, Microtus montanus" elsewhere in the Annual Reports of the UW-NPS Research Center, 2000). The digestive tract (from the pyloric sphincter to the anus) was excised, cleaned of all attached mesenteries and washed of its contents. It was then processed as described by Lochmiller et al. (1992), pinned flat under acetic acid and pressed flat between two sheets of acetate for one week. Outlines of the gut and the location of all lymphoid follicles were then traced onto the acetate.

The surface area of the gut and of all the lymphoid tissue was determined as follows. The acetate tracings were placed on a digitizing palette. The outline of the three segments of the gut (small intestine, SI; cecum, CE; large intestine, LI) and the outlines of all the lymphoid aggregates were traced with a digitizing mouse, converting the outlines into a digital image, yielding a measurement of these surface areas to the nearest square centimeter.

\section{RESULTS AND DISCUSSION}

The surface area of the gut and the location of all lymphoid patches was determined separately for each of the three major segments of the gut: the small intestine (SI), the large intestine (LI) and the cecum (CE).

Gut-associated lymphoid tissue (GALT), present in all segments of the intestinal tract, occurred as single lymphoid follicles and as aggregates of such follicles (Peyer's patches). The total numbers of GALT patches and their distribution varied considerably among individuals. Nevertheless, certain patches ("universal" patches) not only occurred in all voles, their relative size and location within the gut also 
remained unchanged, regardless of the sex and age of the animals.

There is a total of four such "universal" patches in the gut of $M$. montanus: two in the SI (the duodenal patch and the ileocecal patch), one in the CE (the apical patch) and one in the LI (the colonic patch).

1. The duodenal patch is the first patch the digesta encounter as they enter the gut. It is invariably larger than any of the other lymphoid patches and occurs at the end of approximately the first $20 \%$ of the small intestine. Lymphoid tissue between the pyloric sphincter and the duodenal patch is extremely rare, when present it occurs only as single lymphoid follicles.

2. The ileocecal patch is the last lymphoid patch of the small intestine and is located at the start of the final $6 \%$ of the small intestine. There are never any single lymphoid follicles or lymphoid patches between the ileocecal patch and the ileocecal junction.

3. The apical patch is located in the apex of the cecum. Regardless of the total length of the cecum the apical patch is never more than $6 \mathrm{~mm}$ from the apex. There are never any lymphoid patches or single lymphoid follicles between the apex of the cecum and the apical patch.

4. The colonic patch occurs at the end of the post-cecal spiral, where the striations on the luminal surface of the large intestine disappear. This is located at approximately the end of the first $46 \%$ of the large intestine.

In addition to the above mentioned regions of the gut that were invariably devoid of lymphoid tissue there were others. Lymphoid tissue was never found in the lower $42 \%$ of the cecum, in the ampulla coli and in the first $35 \%$ of the postcecal spiral. Lymphoid tissue was extremely uncommon in the last $12-15 \%$ of the large intestine. In the rare instances where it was found it was invariably limited to single lymphoid follicles.

There was no significant sexual dimorphism in the number of multifollicular lymphoid patches, regardless of the age of the animals. Invariably, the smallest number of patches occurred in the $\operatorname{CE}(x=3.80$, range 2-6) and the largest in the LI ( $x=7.75$, range 512). The number in the SI was intermediate between the $\mathrm{CE}$ and the $\mathrm{LI}(\mathrm{x}=4.88$, range 3-8). Although there were no significant differences between the groups, some trends were evident nevertheless. The smallest total numbers of multifollicular lymphoid patches were found in juveniles; the numbers increased as animals aged (i.e., larger in subadults, largest in adults).
However, the numbers of lymphoid patches in the overwintering adults (i.e., adults trapped in the spring) were smaller than those seen in adults at the end of the summer, although such overwintering adults were older than the adults trapped during the summer. A similar phenomenon has been observed in my laboratory colony (Smith and Pinter 2000). Very old animals have a reduced number of multifollicular patches. However, the loss of multifolllicular patches can be accompanied by an enormous increase in the number of single lymphoid follicles. This phenomenon is not seen in animals from natural populations where single lymphoid follicles are never numerous and occur with the same frequency in all age groups.

Although the numbers of lymphoid patches generally increased with the age of the animals, the same was not true for the total surface area of GALT. Each of the three segments of the gut represents approximately one third of the total gut surface. The SI and the CE account for $35 \%$ each, the total surface of the $\mathrm{LI}$ is $31 \%$. However, GALT is not distributed evenly among these three segments. Two thirds (63\%) of the total GALT surface is located in the SI. The abundance of GALT in the SI is not surprising. In the gut, a thin layer of epithelial cells separates an animal from ingested antigens and pathogens. If the immune system is to respond effectively to antigens in the diet, GALT must sample the digesta for the presence of such antigens as early as possible in their passage through the gut. The smallest GALT surface is in the CE $(13 \%)$; the LI is intermediate with $24 \%$ of the total GALT. Overall, GALT occupies $1.7 \%$ of the total gut surface in the SI, $0.7 \%$ in the $\mathrm{LI}$ and $0.4 \%$ in the CE. This ratio remains remarkably constant in all animals, regardless of their sex or age. As the animals age, however, the total GALT surface (absolute and relative amount) decreases. For example, in juveniles GALT occupies approximately $3 \%$ (and rarely less than $2.5 \%$ ) of the total SI surface. In contrast, GALT in the SI of adult animals is rarely more than $1 \%$ of the total surface. The same relationship holds true for the two other segments of the gut.

As mentioned earlier, the decrease in the total GALT surface is both absolute and relative. Such dynamics in the morphometric characteristics of the gut indicate that in Microtus montanus GALT develops early in life. At weaning, the total surface area of GALT is larger than it will be at any future time in their life. Young animals have a large absolute amount of GALT; however, since their total gut surface is smaller than that of adults, GALT in juveniles also occupies a large relative surface of the gut. This means that, in young voles, there is a particularly efficient sampling of the 
digesta, resulting in an early and efficient mounting of an immune response against antigens in the food. As animals age, the total gut surface increases and the total amount of GALT surface decreases. This translates into a decrease in the relative amount of GALT and a decrease in the efficiency with which antigens in the digesta can be monitored.

In summary, GALT was most abundant in juvenile voles and least abundant in adults. It was most abundant in the SI, a not surprising finding since GALT is a first line defense against dietary antigens and pathogens. There was no sexual dimorphism in any aspect of GALT. There was also no effect of season: juveniles invariably had the largest amount of GALT, regardless of their season of birth.

\section{$\downarrow$ ACKNOWLEDGMENTS}

I gratefully acknowledge the availability of the facilities of the University of Wyoming-National Park Service Research Center, the enthusiastic support of the Research Center staff, and funding from the UWNPS Research Grant Program without which it would have been impossible to accomplish this work. I am deeply grateful to the late Dr. Robert Lochmiller who welcomed me in his lab and gave most unselfishly and generously of his time in teaching me new techniques, some of which were used in this study.

\section{$\uparrow$ LITERATURE CITED}

Li, J., K.A. Kudsk, B. Gocinski, D. Dent, J. Glezaer and B. Langkamp-Henken. 1995. Effects of parenteral and enteral nutrition on gutassociated lymphoid tissue. J. Trauma, 39:4452.
Lochmiller, R.L., M.R. Vestey and S.T. McMurray. 1993. Phenotypic variation in lymphoproliferative responsiveness to mitogenic stimulation in cotton rats. $J$. Mamm., 74:189-197.

Lochmiller, R.L., M/R. Vestey and D. Nash. 1992. Gutassociated lymphoid tissue in the cotton rat (Sigmodon hispidus) and its response to protein restriction. J. Wildl. Dis., 28:1-9.

Lopez, M.C., M.E. Roux, S.H. Langhini, M.E.Rio and J.C. Sanahuja. 1985. Effect of severe protein deficiency and refeeding on precursor IgAcells in Peyer's patches of growing rats. Nutr. Rep. Internat., 32:667-674.

Mihok, S., B.N. Turner and S.L. Iverson. 1985. The characterization of vole population dynamics. Ecol. Monog., 55:399-420.

Pinter, A.J. 1988 Multiannual fluctuations in precipitation and population dynamics of the montane vole, Microtus montanus. Can. J. Zool., 66:2128-2132.

Smith, Z.R. and A.J. Pinter. 2000. Post-weaning development of gut-associated lymphoid tissue (GALT) in Microtus montanus in the laboratory. Poster presented at the $80^{\text {th }}$ Ann. Meeting of the Amer. Soc. Mammalogists, Univ. of New Hampshire, Durham, NH, 17-21 June. 